Canadian

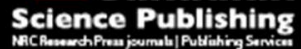

Canadian Journal of Physiology and Pharmacology Revue canadienne de physiologie et pharmacologie

\title{
CLOCK-BMAL1 Regulate the Cardiac L-type Calcium Channel Subunit CACNA1C through the PI3K-Akt Signaling Pathway
}

\begin{tabular}{|r|l|}
\hline Journal: & Canadian Journal of Physiology and Pharmacology \\
\hline Manuscript ID & cjpp-2015-0398.R3 \\
\hline Manuscript Type: & Article \\
\hline Date Submitted by the Author: & 05-Apr-2016 \\
\hline Complete List of Authors: & $\begin{array}{l}\text { Chen, Yanhong; the First Affiliated Hospital, Nanjing Medical University, } \\
\text { Zhu, Didi; the First Affiliated Hospital, Nanjing Medical University, } \\
\text { Yuan, Jiamin; Nanjing Medical University, } \\
\text { Han, Zhonglin; the First Affiliated Hospital, Nanjing Medical University, } \\
\text { Wang, Yao; the First Affiliated Hospital, Nanjing Medical University, } \\
\text { Qian, Zhiyong; the First Affiliated Hospital, Nanjing Medical University, } \\
\text { Hou, Xiaofeng; the First Affiliated Hospital, Nanjing Medical University, } \\
\text { Wu, Tingting; the First Affiliated Hospital, Nanjing Medical University, } \\
\text { Zou, Jian-Gang; the First Affiliated Hospital, Nanjing Medical University, }\end{array}$ \\
\hline Keyword: & $\begin{array}{l}\text { L-type calcium currents, cardiomyocyte action potentials, PI3K-Akt } \\
\text { pathway, ventricular arrhythmia, circadian rhythm }\end{array}$ \\
\hline &
\end{tabular}




\section{CLOCK-BMAL1 Regulate the Cardiac L-type Calcium}

\section{Channel Subunit CACNA1C through PI3K-Akt Signaling}

\section{Pathway}

Chen Yanhong ${ }^{1,2^{*}} ;$ Zhu Didi $^{1 *} ;$ Yuan Jiamin ${ }^{1 *} ;$ Han Zhonglin ${ }^{1 *} ;$ Wang Yao ${ }^{1}$; Qian Zhiyong ${ }^{1}$; Hou Xiaofeng ${ }^{1}$; Wu Tingting ${ }^{1}$; Zou Jian-Gang ${ }^{1}$;

${ }^{1}$ Department of Cardiology, the First Affiliated Hospital, Nanjing Medical University, Nanjing, China;

${ }^{2}$ Department of Cardiology, Wuhan Asia Heart Hospital, Hubei, China Corresponding author: Zou Jian-Gang at Department of Cardiology, the First Affiliated Hospital, Nanjing Medical University, Nanjing, China, jgzou@njmu.edu.cn, phone number: +8602586862862 , fax number: +8602586862862 .

*These authors contributed equally to this work

Pages: 27

Illustrations: 5 


\section{Abstract}

The heterodimerized transcription factors CLOCK-BMAL1 regulate the cardiomyocyte circadian rhythms. The L-type calcium currents play important role in the cardiac electrogenesis and arrhythmogenesis. Whether and how the CLOCK-BMAL1 regulate the cardiac L-type calcium channels are yet to be determined. The functions of the L-type calcium channels were evaluated with patch clamping techniques. Recombinant adenoviruses of CLOCK and BMAL1 were used in the expression experiments. We reported that the expressions and functions of CACNA1C (the a -subunit of the L-type calcium channels) showed circadian rhythms, with the peak at zeitgeber time $3(\mathrm{ZT} 3)$. The endocardial action potential durations90 (APD90) were correspondingly longer at ZT3. The protein levels of the phosphorylated Akt at threonine 308 (pAkt T308) also showed circadian rhythms. Overexpressions of CLOCK-BMAL1 significantly reduced the levels of CACNA1C while increasing the levels of pAkt T308 and pik3r1. Furthermore, the inhibitory effects of CLOCK-BMAL1 on CACNA1C could be abolished by the Akt inhibitor MK2206 or the PDK1 inhibitor GSK2334470. Collectively, our findings suggested that the expressions of the cardiac CACNA1C were under the CLOCK-BMAL1 regulation, probably through the PI3K-Akt signal pathway.

Keywords: L-type calcium currents; cardiomyocyte action potentials; PI3K-Akt pathway; ventricular arrhythmia; circadian rhythm; CLOCK; BMAL1 


\section{Introduction}

The incidence of ventricular arrhythmias (VA) and sudden cardiac death (SCD) exhibit diurnal rhythms, with the well-known morning peaks(Arntz et al. 1993; Muller et al. 1987; Willich et al. 1987). Clinical investigations showed that the time of day was an independent risk factor of ventricular tachycardia (VA) and sudden cardiac death (SCD), being irrelevant to myocardial ischemia (Cohen et al. 1997; Englund et al. 1999), congestive heart failure (Behrens et al. 1997), myocardial hypertrophy (Zehender et al. 1992), initial cardiac rhythm (Thakur et al. 1996), age(Thakur et al. 1996), sex (Savopoulos et al. 2006; Thakur et al. 1996), and the usage of class I (Peters et al. 1994)/class III (Behrens et al. 1997) antiarrhythmic agents. Thus, understanding the circadian control of cardiac electrophsiology is crucial in the treatment of VA/SCD.

The cardiac clock system controls the circadian rhythms of the cardiomyocyte functions. It composes of self sustained feed-back loops of transcription factors(Young 2009), with the cycle length of approximately 24 hours. Among them, CLOCK and BMAL1 are two master factors playing the central roles. CLOCK and BMAL1 form heterodimers CLOCK-BMAL1 before binding to the promoter regions of the target genes to enhance the gene transcriptions (Asher and Schibler 2011; Rey et al. 2011; Zhang and Kay 2010). Over the recent years, there have been accumulating evidences suggesting the circadian controls of the cardiac ion channels (Collins and Rodrigo 2010; Jeyaraj et al. 2012; Schroder et al. 2013; Yamashita et al. 2003). Disruptions of the CLOCK-BMAL1 functions have been reported to cause VA 
and SCD(Jeyaraj et al. 2012; Schroder et al. 2013).

As one of the major factors in the cardiac electrogenesis and arrhythmogenesis(Hofmann et al. 2014; Qu et al. 2013; Shaw and Colecraft 2013), the L-type calcium channels have been reported to show circadian rhythms (Collins and Rodrigo 2010; Collins et al. 2013; Ko et al. 2010). However, the relationship between L-type calcium channels and CLOCK-BMAL1 heterodimers is still unknown. In this study, we aimed to explore the regulation of CLOCK-BMAL1 on the cardiac L-type calcium channels and the possible mechanisms.

\section{Materials and Methods}

\section{Circadian entrainment and cardiomyocyte isolation}

All experiments were performed in accordance with the animal care protocols approved by the Nanjing Medical University Institutional Animal Care and Use Committee. Adult guinea pigs (200-500g) were housed under $12 \mathrm{~h} / 12 \mathrm{~h}$ light/dark cycles for two weeks before the experiments. The light was turned on at 7:00 am and off at 7:00 pm. Zeitgeber time 0(ZT0) was defined as the time the light was turned on, and ZT12 as the time off.

The left ventricular endocardial myocytes of guinea pigs were isolated using the methods described previously (Wang et al. 2012; Wang et al. 2009; Wang et al. 2015), at predetermined time-points. The free endocardial myocytes were collected and kept in $4^{\circ} \mathrm{C} \mathrm{KB}$ solutions for 1 hour before the patch clamping experiments. All the ion channel currents were recorded within 6 hours after the cardiomyocyte isolation. 


\section{Electrophysiological Recordings}

The single pipette whole cell patch clamping technique was applied to record the action potential durations (APDs) and the ion channel currents. The pipettes had resistances of $1-3 \mathrm{M} \Omega$ after filled with the pipette solutions. The APDs and the currents were measured with the EPC-9 amplifier. The series resistances (Rs) were below $10 \mathrm{M} \Omega$ and were electronically compensated up to $80 \%$. APs were elicited by the injection of a $4 \mathrm{~ms}$ depolarizing pulse through the pipette under the I-Clamp mode, with the cycle length of $1000 \mathrm{~ms}$. APDs were elicited and recorder at $37^{\circ} \mathrm{C}$. For the recordings of the L-type calcium currents, the cardiomyocytes were held at $-80 \mathrm{mV}$, before being depolarized to the levels of $-70 \mathrm{mV}$ to $60 \mathrm{mV}$ in $10 \mathrm{mV}$ increments to elicit the inward currents. The depolarization pulse width was 500ms. The L-type calcium currents were recorded under room temperature.

For the recordings of the IKs/IKr tail currents, the cardiomyocytes were held at $-40 \mathrm{mV}$ to inactivate the sodium channel currents. The L-type calcium current was blocked with $10 \mu \mathrm{M}$ nifedipine. The cardiomyocytes were depolarized to the levels of $-40 \mathrm{mV}$ to $60 \mathrm{mV}$ in $10 \mathrm{mV}$ increments before repolarized to $-40 \mathrm{mV}$ to elicit the tail currents. The depolarization pulse width was $4500 \mathrm{~ms}$. The $\mathrm{IKs} / \mathrm{IKr}$ tail currents were measured as dofetilide $(1 \mu \mathrm{M})$ - resistant currents and chromanol 293B $(20 \mu \mathrm{M})-$ resistant currents, respectively.

\section{Solutions and drugs}

The tyrode's solution used for cardiomyocytes isolation was consisted of (in mmol/L): $\mathrm{NaCl} 143, \mathrm{KCl} 5.4, \mathrm{NaH}_{2} \mathrm{PO}_{4}$ 0.25, HEPES 5, Glucose 5.6, $\mathrm{CaCl}_{2} \cdot 2 \mathrm{H}_{2} \mathrm{O} 1.8$, 
$\mathrm{MgCl}_{2} \bullet 6 \mathrm{H}_{2} \mathrm{O} 0.5(\mathrm{PH} 7.3$ with $\mathrm{NaOH}$ ). The $\mathrm{KB}$ solution was consisted of (in mmol/L): $\mathrm{KOH} 85, \mathrm{KCl} 30, \mathrm{KH}_{2} \mathrm{PO}_{4} 30, \mathrm{MgSO}_{4} \cdot 7 \mathrm{H}_{2} \mathrm{O}$ 3, HEPES 10, EGTA 0.5, Taurine 20, Glucose 10, L-glutamine 50 ( $\mathrm{PH} 7.4$ with $\mathrm{KOH})$; The bath solution used for AP /IKs/IKr recordings was consisted of (in mmol/L): $\mathrm{NaCl} 140, \mathrm{KCL} 3.5, \mathrm{CaCl} 2 \cdot 2 \mathrm{H} 2 \mathrm{O}$ 1.5, $\mathrm{MgSO} \cdot 7 \mathrm{H} 2 \mathrm{O}$ 1.4, HEPES $10(\mathrm{PH} 7.4$ with $\mathrm{NaOH})$. The pipette solution for AP /IKs/IKr recordings was consisted of (in mmol/L): $\mathrm{KCl} 140, \mathrm{CaCl}_{2} \cdot 2 \mathrm{H}_{2} \mathrm{O} 1, \mathrm{Na}_{2} \mathrm{ATP}$ 5, creatine phoshate(disodium salt) 5, HEPES 10, $\mathrm{MgCl}_{2} \bullet 6 \mathrm{H}_{2} \mathrm{O} 2$, EGTA 11, PH7.2 with $\mathrm{KOH}$; The bath solution used for AP recordings was consisted of (in mmol/L): $\mathrm{NaCl} 140, \mathrm{KCL} 3.5, \mathrm{CaCl}_{2} \cdot 2 \mathrm{H}_{2} \mathrm{O} 1.5, \mathrm{MgSO}_{4} \cdot 7 \mathrm{H}_{2} \mathrm{O} 1.4$, HEPES 10 (PH 7.4 with $\mathrm{NaOH})$. The pipette solution for L-type calcium current recordings was consisted of (in mmol/L): $\mathrm{CsCl} 130$, TEA-Cl 20, $\mathrm{MgCl}_{2} \bullet 6 \mathrm{H}_{2} \mathrm{O}$ 1, HEPES 1, EGTA 5, MgATP 5, Phosphocreatine Na 5, PH7.2 with CsOH. The bath solution used for L-type calcium current recordings was consisted of (in mmol/L): Choline chloride $120, \mathrm{CaCl}_{2} \cdot 2 \mathrm{H}_{2} \mathrm{O}$ 1.8, $\mathrm{MgCl}_{2} \bullet 6 \mathrm{H}_{2} \mathrm{O} 1$, HEPES 10, $\mathrm{CsCl} 20$, Glucose 10, PH 7.4 with 2M Tris base.

\section{Adult Guinea Pig Cardiomyocyte Culture and Adenovirus Transfection}

Endocardial myocytes were isolated from guinea pig hearts as described previously(Wang et al. 2012; Wang et al. 2009; Wang et al. 2015). The cardiomyocytes were isolated at ZT3. After $1 \mathrm{hr}$ in the $\mathrm{KB}$ solutions, the cardiomyocytes were restored to normal extracellular $\mathrm{Ca}^{2+}$ concentration $(1.8 \mathrm{mM})$, before being plated in the 6 -well plates $\left(10^{5}\right.$ cells/well). The cardiomyocyte culture medium was M199 medium (Hyclone) supplemented with (in mmol/L) HEPES 25, $\mathrm{NaHCO}_{3}$ 26, creatine 5, L-carnitine 2, taurine 5, Insulin-transferrin-selenium 
(Invitrogen) 1\%, penicillin $100 \mathrm{IU} / \mathrm{ml}$, and streptomycin $100 \mathrm{mg} / \mathrm{ml}$. Unless otherwise specified, glucose was present at a concentration of $5 \mathrm{mmol} / \mathrm{L}$. The cardiomyoctes were cultured at $37^{\circ} \mathrm{C}$ under $95 \%$ oxygen and $5 \%$ bicarbonate. The cultured myocytes were obtained from two hearts each time, and the experiments were repeated for three times.

The recombinant adenoviruses pAd CMV-BMAL1-IRES-GFP and pAd CMV-CLOCK-IRES-RFP were purchased from Hanbio (Shanghai, China), labeled with GFP or RFP, respectively. The adenoviruses pAd CMV-IRES-GFP and pAd CMV-IRES-RFP were used as negative controls. The multiplicity of adenovirus infection (MOI) was 20. GFP and RFP were detected under a fluorescence microscope $48 \mathrm{~h}$ after the transfections. In the overexpression experiments, the cardiomyocytes were stored in the KB solution for 1 hour before they were restored to normal extracellular $\mathrm{Ca} 2+$ concentration $(1.8 \mathrm{mM})$. Then the cardiomyocytes were plated in the 6-well plates $\left(105 \mathrm{cells} /\right.$ well) and were cultured at $37^{\circ} \mathrm{C}$ under $95 \%$ oxygen and 5\% bicarbonate. After 2 hours, the culture medium were replaced with mediums containing the recombinant adenoviruses, in which the cardiomyocytes were incubated for 2 hours. After the transfections were completed, the cardiomyocytes were cultured in fresh mediums for 48 hours before the WB and patch clamping were performed. Altogether, it took around 53 hours before the L-type calcium currents and the target protein levels were measured.

\section{Western blotting Analysis}


Samples were prepared and collected every 3 hours starting from ZT0, in a similar fashion as previous studies(Wang et al. 2012). Briefly, left ventricle of the hearts were homogenized in RIPA buffer. The samples were separated on $10 \%$ sodium dodecyl sulfate-polyacrylamide gel electrophoresis (SDS-PAGE) gels and transferred to PVDF membranes. The primary antibodies used in this study were anti-Cav1.2 (Alomone, Jerusalem, Israel) , anti-CLOCK (Abcam), anti-Akt (total Akt) (Cell Signal Technology), anti-pAkt T308(Cell Signal Technology), anti-pAkt S473(Cell Signal Technology), anti- PDK1(Cell Signal Technology) and anti- $\beta$ tubulin(Cell Signal Technology). $\quad \beta$ tubulin was used as the internal reference. All measurements were repeated for at least 3 times.

\section{Data Analysis}

The data were acquired using Pulse +Pulsefit V 8.53 and were expressed as mean \pm SEM. Data comparisons were made through the unpaired Student's t-test or one-way ANOVA followed by Turkey's post hoc test or Dunn's multiple comparison test, depending on whether the groups followed a Gaussian distribution. All the comparisons were made using SPSS16. Differences were considered statistically significant if $P<0.05$. Circadian rhythms of the protein levels were evaluated with the fittings of the cosine function $(Y=$ Amplitude $* \cos ((2 * \mathrm{pi} * X /$ Wavelength $)+$ Phaseshift $)$ + ampshift), using graphpad 5.0. 


\section{Results}

\section{The CACNA1C expressions and functions had circadian rhythms}

The CACNA1C protein levels had significant circadian variations (Figure 1A, 1B), with the peak at ZT3 and the trough at ZT15 $(P<0.05, n=3$ for each time point $)$. ZT3 and ZT15 were chosen for further investigations on the L-type calcium currents and APDs.

After the circadian entrainment, the guinea pig endocardial myocytes were isolated at ZT3 and ZT15, respectively. The L-type calcium currents were significantly larger at ZT3 than ZT15 (Figure 1C, 1D). The average peak L-type calcium current densities (at $10 \mathrm{mV}$ ) were $9.00 \pm 0.52, n=15$ at ZT3 vs. $6.44 \pm 0.57$, $n=18$ at $\mathrm{ZT} 15(P<0.01)$.

To further determine whether the circadian rhythm of the L-type calcium channels affect the APDs, we compared the encocardial APD90s at ZT3 and ZT15. The APD90 were significantly longer at ZT3 $(229.10 \pm 13.96 \mathrm{~ms}$ at ZT3 vs. $184.8 \pm$ $4.19 \mathrm{~ms}$ at $\mathrm{ZT} 15, n=12, P<0.01$ Figure1E). We also measured the major outward potassium currents (IKs and $\mathrm{IKr}$ ) of repolarizing periods and found no significant differences (data not shown).

In another set of experiments, cadiomyocytes were isolated at ZT3 and ZT15 respectively, before they were cultured for 48 hours. Then the CACNA1C expression levels were measured with western blotting. The results showed that the difference in whole-heart CACNA1C expression is still observable in the cultured cardiomyocytes, that the CACNA1C expressions were higher in cultured 
cardiomyocytes isolated at ZT 3 than at ZT15. The sample traces and quantifications were compared in Figure 1G,1H.

\section{The phosphorylated Akt (pAkt T308) levels showed circadian rhythms}

Next, we investigated the circadian rhythms of the Akt and PDK1 levels. The CLOCK protein levels were also measured as controls (Figure 2B, ). The results showed that pAkt T308 exhibited strong circadian fluctuations (Figure 2C) $(P<0.01)$, with the peak at ZT9. The levels of PDK1 and total Akt mildly fluctuated in the diurnal cycles, but the differences did not reach the statistic significance (Figure 2D, Figure 2E).

3. Overexpressions of CLOCK-BMAL1 affected the expressions of the phosphorylated Akt, pike3r1 and CACNA1C.

We cotransfected the cultured cardiomyocytes with CLOCK and BMAL1 recombinant adenoviruses (Figure 3A) and measured the protein expressions of pAkt and CACNA1C.

Compared with the control, the co-expressions of both CLOCK and BMAL1 upregulated pAkt T308 but downregulated CACNA1C. The Overexpressions of CLOCK or BMAL1 alone did not affect pAkt T308 or CACNA1C levels (Figure 3B). Phosporylated PDK1 (pPDK1) and pik3r1 levels were measured in the CLOCK/BMAL1 over-expressed cardiomyocytes. The results showed that pik3r1, but not pPDK1 level, was higher in the CLOCK+BMAL1 over-expressed cardiomyocytes

(Figure 3B). The quantification of the targeted protein expressions were presented in Figure 3C. The level of pik3r1 was increased by 2.14 folds in CLOCK+BMAL1 
over-expressed cardiomyocytes compared to the control $(P<0.05)$ Also, CLOCK-BMAL1 improved the pAkt T308 levels for about 2.4 folds averagely $(P<0.05)$, while CACNA1C expressions were decreased for about $45 \%(P<0.0)$..

The L-type calcium currents were significantly inhibited in the CLOCK+BMAL1 overexpression group $(P<0.05)$. The peak current densities were significantly smaller in the CLOCK+BMAL1 group $(1.41 \pm 0.17$ in CLOCK+BMAL1 group vs. $3.22 \pm 0.53$ in the control group, Figure $4 \mathrm{C}, P<0.05, n=5$ for each group). CLOCK or BMAL1 alone did not affect the L-type calcium currents. The peak current densities were $3.46 \pm 0.97$ in the CLOCK group and $3.34 \pm 0.58$ in the BMAL1 group. Figure 4A showed the sample traces of the L-type calcium currents. Figure 4B showed the I-V curves of the L-type calcium currents ( $n=5$ for each group).

\section{Inhibitions of Akt or PDK1 abolished the inhibitory effects of CLOCK-BMAL1 on the L-type calcium channels}

We hypothesized that the circadian clock might affect the expressions of CACNA1C through the PI3K-Akt signal pathway. To testify the hypothesis, we used the Akt inhibitor MK2206 (1uM in the medium) or the PDK1 inhibitor GSK2334470 ( $1 \mathrm{uM}$ in the medium). The two agents were added into the culture mediums after the transfections of the adenoviruses. The protein expressions and functions of the four groups were evaluated $48 \mathrm{~h}$ thereafter.

Figure 5A showed the protein expressions. The left panel was the sample traces of CLOCK, pAkt T308 and CACNA1C. The right panel of figure 5A showed the quantification of the targeted protein levels. CLOCK-BMAL1 improved the pAkt 
T308 levels for about 2.4 folds averagely $(P<0.05)$, while CACNA1C expressions were decreased for about $45 \%(P<0.05)$. The effects of CLOCK-BMAL1 on the CACNA1C expression could be reversed by either MK2206 (1uM) or GSK 2334470 (1uM).

The I-V curves were presented in the left panel of figure 5C, The L-type calcium currents were significantly inhibited by CLOCK-BMAL1 $(P<0.05, n=5$ for each group), which could be reversed with the Akt inhibitor MK2206 (1uM) or the PDK1 inhibitor GSK2334470 (1uM). The right panel of figure 5C compared the peak current densities. The peak current densities were significantly smaller in the CLOCK+BMAL1 group $(1.41 \pm 0.17$ in CLOCK+BMAL1 group vs. $3.22 \pm 0.53$ in the control group $P<0.05, n=5$ for each group). The peak current densities were $3.41 \pm 1.09$ in the MK2206 treated CLOCK+BMAL1 group and $3.46 \pm 0.97$ in the GSK2334470 treated group. Both were not significantly different from the control.

To rule out the possible effects of MK2206 and GSK2334470 on the L-type calcium currents. We performed an additional set of experiments. The cultured cardiomyocytes were assigned into three groups: control, MK2206 treated group and GSK 2334470 treated group. The MK2206 or GSK 2334470 were added into the culture mediums without adenovirus transfections. After $48 \mathrm{~h}$, we measured the L-type calcium currents. The left panel of figure 5E presented the I-V curves of the three groups. Neither MK2206 nor GSK2334470 affected the L-type calcium channels ( $P>0.05, n=5$ for each group). The right panel of figure 5E compared the peak current densities $(3.32 \pm 0.54$ in the control group, $3.03 \pm 0.47$ in the MK2206 group, $3.25 \pm$ 
0.44 in the GSK2334470 group, $P>0.05, n=5$ for each group). 


\section{Discussion}

In this study we reported that CLOCK-BMAL1 heterodimers regulated the expressions and functions of the cardiac L-type calcium channel subunit CACNA1C, and that CLOCK-BMAL1 might exert the regulations through the PI3K-Akt signal pathway.

The cardiac circadian clock system are composed of a set of transcription factors, which form negative feedback loops. The heterodimers CLOCK-BMAL1 act as core factors (Asher and Schibler 2011; Durgan and Young 2010). The disruption of the cardiac clock system (BMAL1 knockout) caused VA and SCD (Jeyaraj et al. 2012; Schroder et al. 2013), indicating the important role of the cardiac CLOCK-BMAL1 in the onset of VA/SCD. Whether the CLOCK-BMAL1 heterodimers regulate the L-type calcium channels was still a question to answer.

The reports on the circadian variations of the L-type calcium currents varied in the previous studies. Collins et al. reported that L-type calcium currents in rats were larger at ZT15(Collins et al. 2013). Ko, Shi et al. reported the peak of L-type calcium currents in embryo chick hearts was reached at ZT17-20 (Ko et al. 2010). Ko et al. also reported that the mRNA levels and protein expressions of VGCC $\alpha 1 \mathrm{C}$ remained constant throughout the day in mice ventricles(Ko et al. 2011). We found that the guinea pig cardiomyocyte CACNA1C expressions \& functions peaked at ZT3. The discrepancy was likely caused by some reasons. Firstly, the selected time points varied among these studies. Collins et al. used two time points ( ZT3 and ZT15). Ko et al. measured the protein expressions and the L-type calcium currents every 4 hours 
in the diurnal cycles. We measured the protein expression levels every 3 hours and picked the two time points with the largest expression variations for patch clamping measurements. Although the cardiomyocyte clock system is ever changing in its status, it takes 3-6 hours (average transcription/translation time) to make a measurable difference. Thus, we think that $3-4 \mathrm{hr}$ time intervals are appropriate to evaluate the circadian rhythms of a target gene, and long intervals might miss the actual peak/trough of the gene expressions. Secondly, there were discrepancies in the electrophysiology recording protocols. Some experiments used acutely-isolated cardiomyocytes(Collins and Rodrigo 2010; Collins et al. 2013), while others used cardiomyocytes cultured overnight (Ko et al. 2010). It was reported that the cardiomyocyte clock was ticking on under cell culture conditions(Durgan et al. 2005), thus cell culturing might distort the results. Another reason was the differences in the animal circadian behavioral phenotypes. The study by Collins et al using acute myocyte preparation found the peak of the L-type calcium current 12 hours later than ours(Collins and Rodrigo 2010). The defferences in the animal circadian behavior should be taken into account. Collins et al. used rats as animals while we used guinea pigs. When rats are nocturnal animals, guinea pigs have very fragmented sleep patterns. They are usually active throughout the light and dark phases and the circadian behavior varies among different individuals(Akita et al. 2001; Lee et al. 2014). Last bu not the least, there were the species difference. The published reports on the circadian rhythms of the L-type calcium channels have used different animal models: rats, mice and chick embryos. The genes coding for L-type calcium channels 
are very different in chick embryos from rats/mice. The guinea pigs also had unique characteristics in the cardiomyocyte electrophysiologies, like the lack of the Ito currents and the large IKr currents. The circadian regulations on the cardiac calcium channels might be different among different species as well.

It was interesting to discover the strong circadian fluctuations of the activated Akt. We also measured the protein levels of PDK1, whose circadian variations did not reach the statistical significances. What was clear though, was the fact that CLOCK-BMAL1 could induce the Akt phosphorylations. How the inductions were accomplished called for further investigations. Akt is phosphorylated by PDK1 at threonine 308. However, PDK1 alone could not accomplish the Akt phosphorylation. Akt binds to PIP3/PIP2 produced by PI3K at the plasma membrane. The binding of PIP2/PIP3 produces a conformational change, which is crucial for the Akt phosphorylation. Besides, the phosphorylation needs the co-localization of Akt and PDK1 on the cell memberane, which was completed through their bindings to PIP2/PIP3. Thus, PIP2/PIP3 plays a pivotal role in the Akt phosphorylation(Gagliardi et al. 2015). Recent researches have revealed that pik3r1 was under direct regulation of cardiac BMAL1(Young et al. 2014). Pik3r1 encodes for $\mathrm{p} 85 \alpha$, the regulatory subunit of PI3K, which act crucial in the Akt phosphorylation. Also, the authors reported that the levels of AKT T308 were lower in cardiac-specific-BMAL1-knockout mice hearts, which was consistent with our findings. Zhang et al. reported that Akt phosphorylation was regulated by BMAL1 in mice liver (Zhang et al. 2014), and the authors observed RIZTOR, the key 
component of the mTORC2 complex, might act critically in the BAML1 inductions of the Akt phosphorylation. Our results showed that pik3r1 could be induced by CLOCK-BMAL1 dimers, which was consistent with previous reports by Yound et.al. Since CLOCK-BMAL1 were transcription factors, it took 6-8hrs for the transcriptions and translations to take place. Thus, it was reasonable that the effects of the CLOCK-BMAL1 reached the peak with a delay.

The PI3K-Akt pathway influences the protein synthesis/degradation, as well as the ion channel trafficking and translocation. Previous studies proved that PI3K modulated the L-type calcium channels and played a crucial role in vascular excitation-contraction coupling(Le Blanc et al. 2004). Huang et al. reported that mTORC1 signaling played a role in the circadian regulations of the L-type calcium channels, in part through its regulation of ion channel trafficking and translocation(Huang et al. 2013).Our findings further supported that the circadian control of the CACNA1C was accomplished through the PI3K-Akt pathway.

On the other hand, L-type calcium channels are widely expressed, and they are under complex and tissue-specific regulations. In the SCN regions, the CACNA1C expressions were inhibited by the nuclear factor REV-ERBa1, which was another core circadian factor (Schmutz et al. 2014). In the brain arterial myocytes, $\mathrm{Ca}(\mathrm{V}) 1.2$ channel expression was regulated by NF-kappaB through IP(3)R-mediated SR $\mathrm{Ca}(2+)$ release(Narayanan et al. 2010). L-type calcium channels were also reported to be inhibited by the protein STIMI(Park et al. 2010; Wang et al. 2010). Thus, the circadian regulations on the L type calcium channels are probably complex 
and other pathways should be included in the further investigations.

To investigate the functional roles of the L type calcium current rhythms, we measured the APDs and found corresponding circadian variations. APs are composed by special types of voltage-gated ion channels embedded in the cardiomycyte memberane, of which the L-type calcium channels produce the major inward currents during the phase 2 and 3 repolarization. We also measured the major outward potassium channels (IKs and IKr) and found no significant differences between ZT3 and ZT15 (data not shown). Thus, the circadian variations of the APD90 are supposed to be caused by the circadian rhythms of the L-type calcium channel. Our findings indicated that the CACNA1C circadian rhythms might have impact on the cardiomyocyte repolarization. Further investigations are needed to evaluate the comprehensive impact of CLOCK-BMAL1 on the cardiac electrophysiology and arrhythmogenesis.

To conclude, we identified CLOCK-BMAL1 as novel regulators of the cardiac calcium channels. CLOCK-BMAL1 regulated the expressions and functions of the cardiac CACNA1C, through the PI3K-AKT pathway. We provide new information on the mechanisms of circadian regulation of heart function. However, further studies on the CLOCK-BMAL1 impacts on the cardiac electrogenesis/arrhythmogenesis should be carried out.

\section{Limitations}

Although our findings highlight CLOCK-BMAL1 as novel regulators of the cardiomyocyte L-type calcium channels, it remains unclear how the CLOCK-BMAL1 
promotes the Akt phosphorylation. Also, when evaluating the functional impacts of the CLOCK-BMAL1 regulations, only endocardial myocytes APDs were investigated. Further studies on the ex-vivo perfused hearts or on the whole hearts in vivo are needed to demonstrate the CLOCK-BMAL1 impacts on the cardiac electrophysiology through its regulations on the CACNA1C .

\section{Declaration of interest:}

The authors report no conflicts of interest.

\section{Acknowledgements:}

This work was supported by grants from the National Natural Science Foundation of China (No. 81170162 and No. 81470457), A Project Funded by the Priority Academic Program Development of Jiangsu Higher Education Institutions (PAPD) and the Graduate Innovation Foundation of Jiangsu Province (JX22013281 and KYLX_0923). 


\section{References:}

Akita, M., Ishii, K., Kuwahara, M., and Tsubone, H. 2001. The daily pattern of heart rate, body temperature, and locomotor activity in guinea pigs. Exp. Anim. 50(5): 409-415.

Arntz, H.R., Willich, S.N., Oeff, M., Bruggemann, T., Stern, R., Heinzmann, A., Matenaer, B., and Schroder, R. 1993. Circadian variation of sudden cardiac death reflects age-related variability in ventricular fibrillation. Circulation, 88(5 Pt 1): 2284-2289.

Asher, G., and Schibler, U. 2011. Crosstalk between Components of Circadian and Metabolic Cycles in Mammals. Cell Metab. 13(2): 125-137.

Behrens, S., Ney, G., Fisher, S.G., Fletcher, R.D., Franz, M.R., and Singh, S.N. 1997. Effects of amiodarone on the circadian pattern of sudden cardiac death (Department of Veterans Affairs Congestive Heart Failure-Survival Trial of Antiarrhythmic Therapy). Am. J. Cardiol. 80(1): 45-48.

Cohen, M.C., Rohtla, K.M., Lavery, C.E., Muller, J.E., and Mittleman, M.A. 1997. Meta-analysis of the morning excess of acute myocardial infarction and sudden cardiac death. Am. J. Cardiol. 79(11): 1512-1516.

Collins, H.E., and Rodrigo, G.C. 2010. Inotropic response of cardiac ventricular myocytes to beta-adrenergic stimulation with isoproterenol exhibits diurnal variation: involvement of nitric oxide. Circ. Res. 106(7): 1244-1252.

Collins, H.E., Turrell, H.E., Samani, N.J., and Rodrigo, G.C. 2013. Diurnal variation in excitation-contraction coupling is lost in the adult spontaneously hypertensive rat heart. J. Hypertens. $31(6): 1214-1223$.

Durgan, D.J., Hotze, M.A., Tomlin, T.M., Egbejimi, O., Graveleau, C., Abel, E.D., Shaw, C.A., Bray, M.S., Hardin, P.E., and Young, M.E. 2005. The intrinsic circadian clock within the cardiomyocyte. Am. J. Physiol. 289(4): H1530-1541.

Durgan, D.J., and Young, M.E. 2010. The cardiomyocyte circadian clock: emerging roles in health and disease. Circ. Res. 106(4): 647-658.

Englund, A., Behrens, S., Wegscheider, K., and Rowland, E. 1999. Circadian variation of malignant ventricular arrhythmias in patients with ischemic and nonischemic heart disease after cardioverter defibrillator implantation. European 7219 Jewel Investigators. J. Am. Coll. Cardiol. 34(5): 1560-1568.

Gagliardi, P.A., di Blasio, L., and Primo, L. 2015. PDK1: A signaling hub for cell migration and tumor invasion. Biochim. Biophys. Acta, 1856(2): 178-188.

Hofmann, F., Flockerzi, V., Kahl, S., and Wegener, J.W. 2014. L-type CaV1.2 calcium channels: from in vitro findings to in vivo function. Physiol. Rev. 94(1): 303-326.

Huang, C.C., Ko, M.L., and Ko, G.Y. 2013. A new functional role for mechanistic/mammalian target of rapamycin complex 1 (mTORC1) in the circadian regulation of L-type voltage-gated calcium channels in avian cone photoreceptors. PloS one, 8(8): e73315.

Jeyaraj, D., Haldar, S.M., Wan, X., McCauley, M.D., Ripperger, J.A., Hu, K., Lu, Y., Eapen, B.L., Sharma, N., Ficker, E., Cutler, M.J., Gulick, J., Sanbe, A., Robbins, J., Demolombe, S., Kondratov, R.V., Shea, S.A., Albrecht, U., Wehrens, X.H., Rosenbaum, D.S., and Jain, M.K. 2012. Circadian rhythms govern cardiac repolarization and arrhythmogenesis. Nature, 483(7387): 96-99.

Ko, M.L., Shi, L., Grushin, K., Nigussie, F., and Ko, G.Y. 2010. Circadian profiles in the embryonic chick heart: L-type voltage-gated calcium channels and signaling pathways. Chronobiol. Int. 27(9-10): 1673-1696.

Ko, M.L., Shi, L., Tsai, J.Y., Young, M.E., Neuendorff, N., Earnest, D.J., and Ko, G.Y. 2011. Cardiac-specific mutation of Clock alters the quantitative measurements of physical activities without changing 
behavioral circadian rhythms. J. Biol. Rhythms, 26(5): 412-422.

Le Blanc, C., Mironneau, C., Barbot, C., Henaff, M., Bondeva, T., Wetzker, R., and Macrez, N. 2004. Regulation of vascular L-type Ca2+ channels by phosphatidylinositol 3,4,5-trisphosphate. Circ. Res. 95(3): 300-307.

Lee, K.N., Pellom, S.T., Oliver, E., and Chirwa, S. 2014. Characterization of the guinea pig animal model and subsequent comparison of the behavioral effects of selective dopaminergic drugs and methamphetamine. Synapse, 68(5): 221-233.

Muller, J.E., Ludmer, P.L., Willich, S.N., Tofler, G.H., Aylmer, G., Klangos, I., and Stone, P.H. 1987. Circadian variation in the frequency of sudden cardiac death. Circulation, 75(1): 131-138.

Narayanan, D., Xi, Q., Pfeffer, L.M., and Jaggar, J.H. 2010. Mitochondria control functional CaV1.2 expression in smooth muscle cells of cerebral arteries. Circ. Res. 107(5): 631-641.

Park, C.Y., Shcheglovitov, A., and Dolmetsch, R. 2010. The CRAC channel activator STIM1 binds and inhibits L-type voltage-gated calcium channels. Science, 330(6000): 101-105.

Peters, R.W., Mitchell, L.B., Brooks, M.M., Echt, D.S., Barker, A.H., Capone, R., Liebson, P.R., and Greene, H.L. 1994. Circadian pattern of arrhythmic death in patients receiving encainide, flecainide or moricizine in the Cardiac Arrhythmia Suppression Trial (CAST). J. Am. Coll. Cardiol. 23(2): 283-289.

Qu, Z., Xie, L.H., Olcese, R., Karagueuzian, H.S., Chen, P.S., Garfinkel, A., and Weiss, J.N. 2013. Early afterdepolarizations in cardiac myocytes: beyond reduced repolarization reserve. Cardiovasc. Res. 99(1): 6-15.

Rey, G., Cesbron, F., Rougemont, J., Reinke, H., Brunner, M., and Naef, F. 2011. Genome-wide and phase-specific DNA-binding rhythms of BMAL1 control circadian output functions in mouse liver. PLoS Biol. 9(2): e1000595.

Savopoulos, C., Ziakas, A., Hatzitolios, A., Delivoria, C., Kounanis, A., Mylonas, S., Tsougas, M., and Psaroulis, D. 2006. Circadian rhythm in sudden cardiac death: a retrospective study of 2,665 cases. Angiology, 57(2): 197-204.

Schmutz, I., Chavan, R., Ripperger, J.A., Maywood, E.S., Langwieser, N., Jurik, A., Stauffer, A., Delorme, J.E., Moosmang, S., Hastings, M.H., Hofmann, F., and Albrecht, U. 2014. A specific role for the REV-ERBalpha-controlled L-Type Voltage-Gated Calcium Channel CaV1.2 in resetting the circadian clock in the late night. J. Biol. Rhythms, 29(4): 288-298.

Schroder, E.A., Lefta, M., Zhang, X., Bartos, D.C., Feng, H.Z., Zhao, Y., Patwardhan, A., Jin, J.P., Esser, K.A., and Delisle, B.P. 2013. The Cardiomyocyte Molecular Clock, Regulation of Scn5a and Arrhythmia Susceptibility. Am. J. Physiol. 304(10): C954-65.

Shaw, R.M., and Colecraft, H.M. 2013. L-type calcium channel targeting and local signalling in cardiac myocytes. Cardiovasc. Res. 98(2): 177-186.

Thakur, R.K., Hoffmann, R.G., Olson, D.W., Joshi, R., Tresch, D.D., Aufderheide, T.P., and Ip, J.H. 1996. Circadian variation in sudden cardiac death: effects of age, sex, and initial cardiac rhythm. Ann. Emerg. Med. 27(1): 29-34.

Wang, H., Chen, Y., Zhu, H., Wang, S., Zhang, X., Xu, D., Cao, K., and Zou, J. 2012. Increased response to beta(2)-adrenoreceptor stimulation augments inhibition of $\mathrm{IKr}$ in heart failure ventricular myocytes. PloS one, 7(9): e46186.

Wang, S., Xu, D.J., Cai, J.B., Huang, Y.Z., Zou, J.G., and Cao, K.J. 2009. Rapid component I(Kr) of cardiac delayed rectifier potassium currents in guinea-pig is inhibited by alpha(1)-adrenoreceptor activation via protein kinase A and protein kinase C-dependent pathways. Eur. J. Pharmacol. 608(1-3): 1-6.

Wang, Y., Deng, X., Mancarella, S., Hendron, E., Eguchi, S., Soboloff, J., Tang, X.D., and Gill, D.L. 2010. 
The calcium store sensor, STIM1, reciprocally controls Orai and CaV1.2 channels. Science, 330(6000): 105-109.

Wang, Y., Yuan, J., Qian, Z., Zhang, X., Chen, Y., Hou, X., and Zou, J. 2015. $\beta 2$ adrenergic receptor activation governs cardiac repolarization and arrhythmogenesis in a guinea pig model of heart failure. Sci. Rep. 5: 7681.

Willich, S.N., Levy, D., Rocco, M.B., Tofler, G.H., Stone, P.H., and Muller, J.E. 1987. Circadian variation in the incidence of sudden cardiac death in the Framingham Heart Study population. Am. J. Cardiol. 60(10): 801-806.

Yamashita, T., Sekiguchi, A., Iwasaki, Y.K., Sagara, K., linuma, H., Hatano, S., Fu, L.T., and Watanabe, H. 2003. Circadian variation of cardiac K+ channel gene expression. Circulation, 107(14): 1917-1922.

Young, M.E. 2009. Anticipating anticipation: pursuing identification of cardiomyocyte circadian clock function. J. Appl. Physiol. 107(4): 1339-1347.

Young, M.E., Brewer, R.A., Peliciari-Garcia, R.A., Collins, H.E., He, L., Birky, T.L., Peden, B.W., Thompson, E.G., Ammons, B.J., Bray, M.S., Chatham, J.C., Wende, A.R., Yang, Q., Chow, C.W., Martino, T.A., and Gamble, K.L. 2014. Cardiomyocyte-Specific BMAL1 Plays Critical Roles in Metabolism, Signaling, and Maintenance of Contractile Function of the Heart. J. Biol. Rhythms, 29(4): 257-276.

Zehender, M., Meinertz, T., Hohnloser, S., Geibel, A., Gerisch, U., Olschewski, M., and Just, H. 1992. Prevalence of circadian variations and spontaneous variability of cardiac disorders and ECG changes suggestive of myocardial ischemia in systemic arterial hypertension. Circulation, 85(5): 1808-1815.

Zhang, D., Tong, X., Arthurs, B., Guha, A., Rui, L., Kamath, A., Inoki, K., and Yin, L. 2014. Liver Clock Protein BMAL1 Promotes de Novo Lipogenesis through Insulin-mTORC2-AKT Signaling. J. Biol. Chem. 289(37): 25925-25935.

Zhang, E.E., and Kay, S.A. 2010. Clocks not winding down: unravelling circadian networks. Nature reviews. Mol. Cell Biol. 11(11): 764-776. 


\section{Figure legends}

Figure1. CACNA1C expression, L-type calcium currents, and APD90s showed circadian rhythms.

A. Guinea pig cardiac CACNA1C expression had circadian rhythms, with the peak at ZT3 and the trough at ZT15 $(P<0.05, n=3)$.

B. Sample traces of the CACNA1C protein levels measured with 3 h-intervals in the circadian cycles, starting from ZT0.

C. The I-V curves of the L-type calcium currents at ZT3 and ZT15. The current densities at $10 \mathrm{mV}$ were significantly larger at ZT3 than ZT15 $(9.00 \pm 0.52, \mathrm{n}=15$ at $\mathrm{ZT} 3$ vs. $6.44 \pm 0.57, n=18$ at $\mathrm{ZT} 15)$ $(P<0.01)$.

D. Sample traces of L-type calcium currents at ZT3 and ZT15. The L-type calcium currents were elicited at room temperature. For the recordings of the L-type calcium currents, the cardiomyocytes were held at $-80 \mathrm{mV}$, before depolarized to the levels of $-70 \mathrm{mV}$ to $60 \mathrm{mV}$ in $10 \mathrm{mV}$ increments to elicit the calcium currents, with the pulse width of $500 \mathrm{~ms}$.

E. Comparisons of APD90s between ZT3 and ZT15. The APD90 were longer at ZT3 ( $229.10 \pm 13.96 \mathrm{~ms}$ at $\mathrm{ZT} 3$ vs. $184.8 \pm 4.19 \mathrm{~ms}$ at $\mathrm{ZT} 15, n=12, P<0.01$ ).

F. The representative traces of the APs at ZT3 and ZT15. APs were elicited by the injection of a $4 \mathrm{~ms}$ depolarizing pulse through the pipette. All the APs were recorded at $37 \square$ in the bath solutions, at the cycle length of $1000 \mathrm{~ms}$.

G. Sample traces of the myocyte CACNA1C. Cardiomyocytes were isolated at ZT3 and ZT15 before they were cultured for 48 hours.

H. Comparison of trace-quantifications. CACNA1C levels were higher in cardiomyocytes isolated at ZT3 than in cardiomyocytes isolated at ZT15. 
Figure2. The circadian expressions of the CLOCK, pAkt, total Akt and PDK1

A. Sample traces of the CLOCK, pAkt T308, total Akt and PDK1 measured with 3h-intervals, starting from ZT0 in the diurnal cycles.

B. The CLOCK expressions showed circadian rhythms. The peaks were reached at ZT0 and the troughs at ZT12. The differences were statistically significant $(P<0.05, n=3$ for each time-point). C. The Akt phosphorylation at threonine 308 showed circadian rhythms. The peaks were reached at ZT9 and the troughs at ZT21. The differences were statistically significant $(P<0.05, n=3$ for each time-point).

D. The total Akt levels showed mild circadian fluctuations, but the differences did not reach statistical significance ( $P>0.05, n=3$ for each time-point).

E. The PDK1 expressions showed mild circadian fluctuations, but the differences did not reach the statistical significance ( $P>0.05, n=3$ for each time-point). 
Figure3. CLOCK-BMAL1 over-expressions affected the expressions of pAkt T308 and CACNA1C.

A. CLCOK and BMAL1 recombinant-adenovirus transfected cardiomyocytes under a fluorescence microscope (Nikon; magnification, x200). The transfection efficiencies were observed under fluorescence microscopes. The CLOCK recombinant adenoviruses were labeled with RFP (red fluorescence) and the BMAL1 recombinant adenoviruses with GFP (green fluorescence). The red and green fluorescence could be merged into yellow fluorescence.

B. The sample traces of CLOCK, pAkt T308, CACNA1C, pPDK1 and pik3r1 with $\beta$-tubulin as the internal references. Compared with the control, the co-expressions of both CLOCK and BMAL1 upregulated pAkt T308 and pik3r1 but downregulated CACNA1C. The level of pPDK1 was not affected by CLOCK-BMAL1 over-expression.

C. The quantification of the targeted proteins. CLOCK-BMAL1 increased the pAkt T308 levels for about 2.4 folds averagely $(P<0.05)$. CACNA1C expressions were decreased for about $45 \%$ $(P<0.05)$. The level of pik3r1 was increased by 2.14 folds in CLOCK+BMAL1 over-expressed cardiomyocytes compared to the control $(P<0.05)$. 
Figure4. L-type calcium currents were reduced in the CLOCK-BMAL1 over-expressed cardiomyocytes.

A. L-type calcium currents sample traces of the control and the CLOCK+BMAL1 overexpression group.

B. The I-V curves of the L-type calcium currents of the four groups. The L-type calcium currents were significantly inhibited in the CLOCK+BMAL1 overexpression group $(P<0.05, n=5$ for each group). Compared with the control, CLOCK or BMAL1 alone did not affect the L-type calcium currents. ( $n=5$ for each group)

C. The L-type calcium currents of cultured cardiomyocytes peaked at $0 \mathrm{mV}$. Compared with the control, the peak current densities were significantly smaller in the CLOCK+BMAL1 group (1.41 \pm 0.17 in CLOCK+BMAL1 group vs $3.22 \pm 0.53$ in the control group., $P<0.05, n=5$ for each group). There were no statistical differences among the control, the CLOCK, and the BMAL1 groups.

Abbreviations: C, CLOCK; B; BMAL1. 
Figure5. The inhibitory effects of CLOCK-BMAL1 on the L-type calcium currents were abolished by Akt inhibitor MK2206 or PDK1 inhibitor GSK2334470.

A. The protein expressions of the four groups: control group, CLOCK+BMAL1 transfection group, MK2206 treated CLOCK+BMAL1 transfection group, and GSK2334470 treated CLOCK+BMAL1 transfection group. The left panel was the sample traces of CLOCK, pAkt T308 and CACNA1C. Compared with the control, the co-expressions of both CLOCK and BMAL1 upregulated pAkt T308 but downregulated CACNA1C. The effects of CLOCK-BMAL1 were almost completely reversed by the Akt inhibitor MK2206 or the PDK1 inhibitor GSK2334470. The quantification of the targeted protein levels were presented in the right panel.

B. Sample traces of L-type calcium currents.

C. The left panel showed the I-V curves of the four groups. The L-type calcium currents were significantly inhibited by CLOCK-BMAL1, which could be reversed with the Akt inhibitor MK2206 or the PDK1 inhibitor GSK2334470. The L-type calcium currents peaked at 0mV in the cultured cardiomyocytes. The right panel compared the peak currents. The peak currents were significantly smaller in the CLOCK+BMAL1 group. The MK2206 or GSK2334470 treated groups were not significantly different from the control. The current densities were $1.46 \pm 0.17$ in the CLOCK+BMAL1 group, $3.41 \pm 0.55$ in the MK2206 treated CLOCK+BMAL1 group, $3.46 \pm 0.97$ in the GSK2334470 treated CLOCK+BMAL1 group ( $n=5$ for each group).

D. Sample traces of L-type calcium currents.

E. The left panel showed the I-V curves. There were no significant differences among the control, the MK2206 treated and the GSK2334470 treated group. The right panel compared the peak currents. There were no significant differences among the control, the MK2206 treated and the GSK2334470 treated group. The current densities were $1.46 \pm 0.17$ in the control group, $3.41 \pm 0.55$ in the MK2206 treated group, 3.46 \pm 0.97 in the treated CLOCK+BMAL1 group ( $P>0.05, n=5$ for each group).

Abbreviations: C, CLOCK; B; BMAL1. MK: MK2206; GSK: GSK2334470. 
A

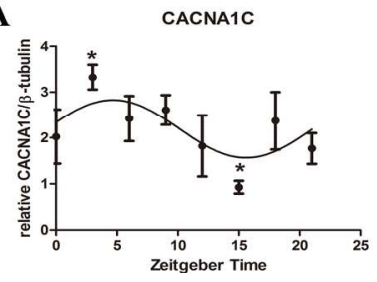

C
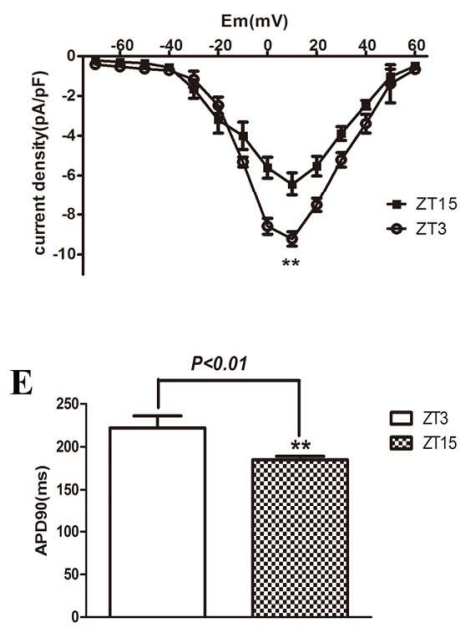

G

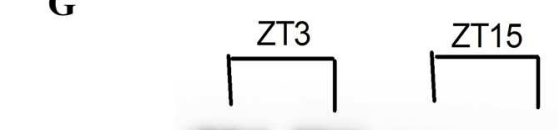

CACNA1C

$\beta$-tubulin
B

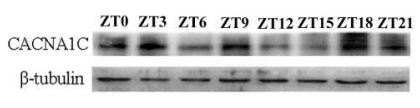

D
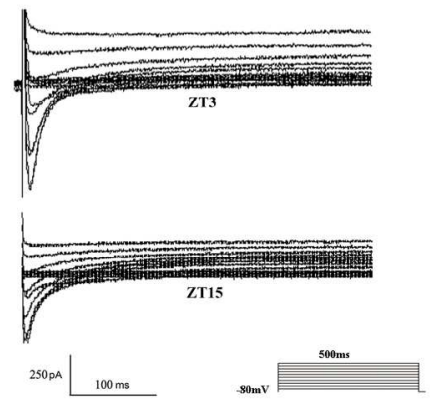

F

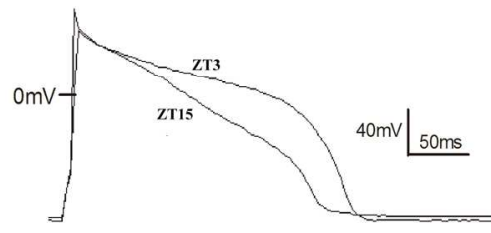

$\mathbf{H}$

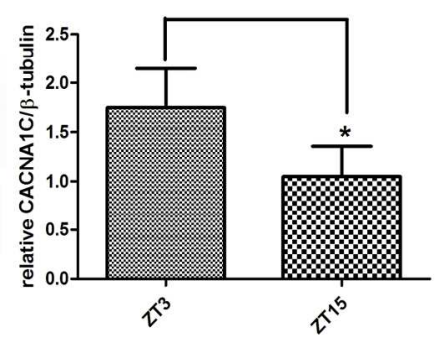

Figure1. CACNA1C expression, L-type calcium currents, and APD90s showed circadian rhythms. $146 \times 211 \mathrm{~mm}(300 \times 300 \mathrm{DPI})$ 

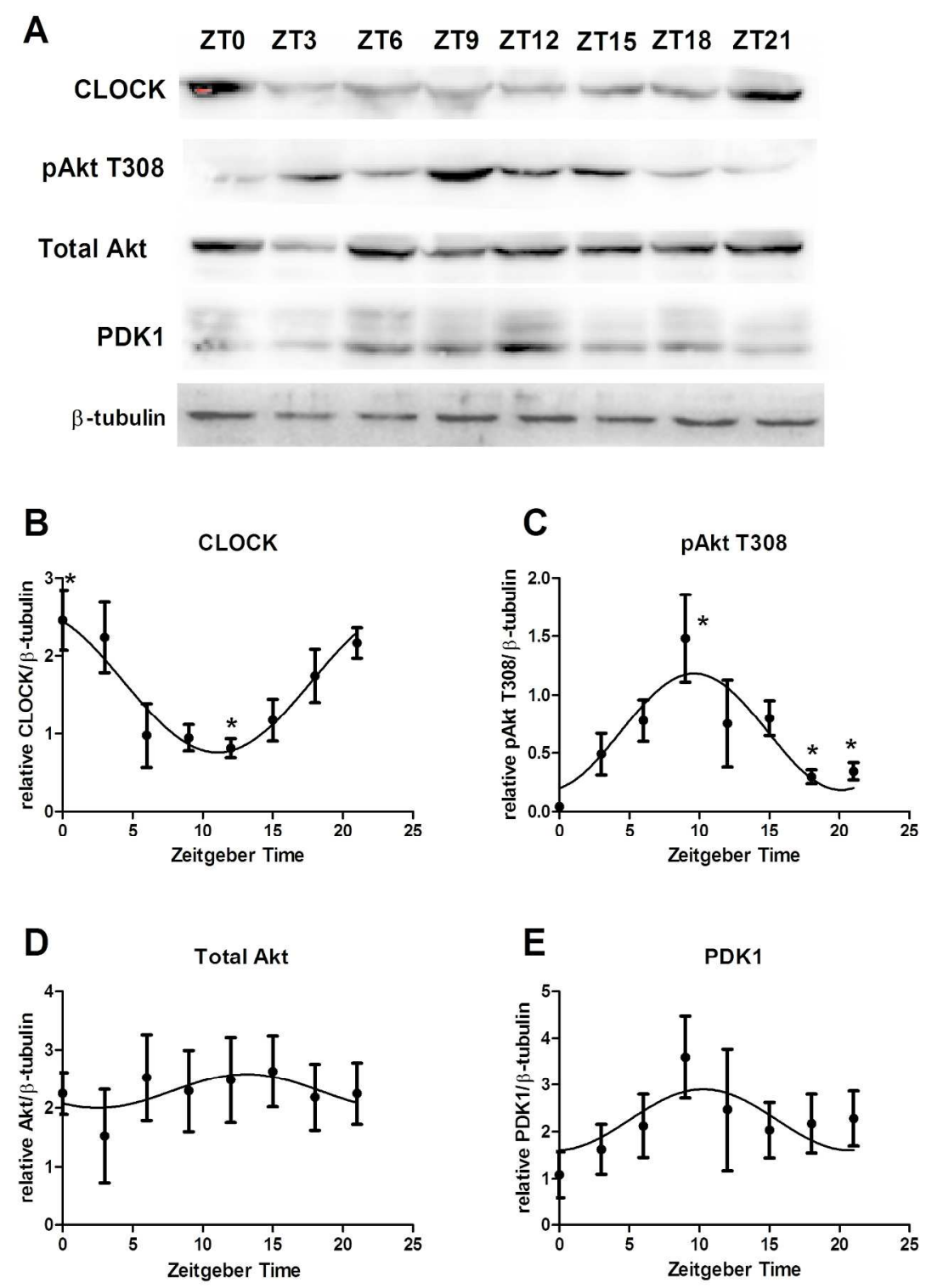

Figure2. The circadian expressions of the CLOCK, pAkt, total Akt and PDK1 $190 \times 251 \mathrm{~mm}(300 \times 300 \mathrm{DPI})$ 
A

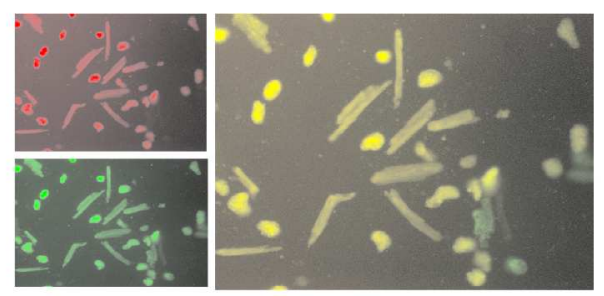

B

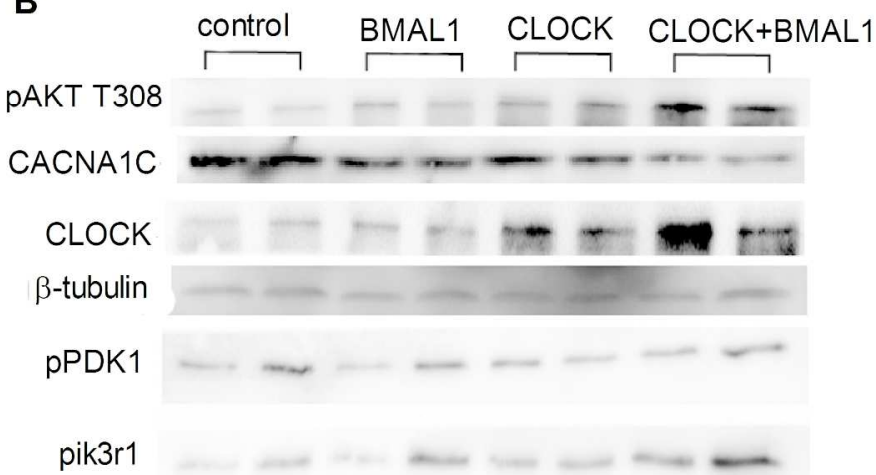

$\beta$-tubulin

C

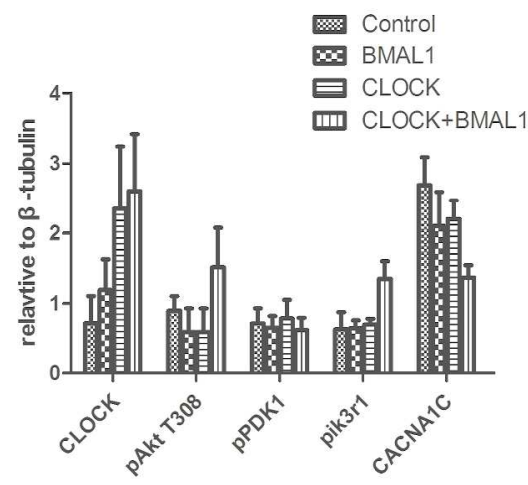

Figure3. CLOCK-BMAL1 over-expressions affected the expressions of pAkt T308 and CACNA1C. $200 \times 281 \mathrm{~mm}(300 \times 300 \mathrm{DPI})$ 
A
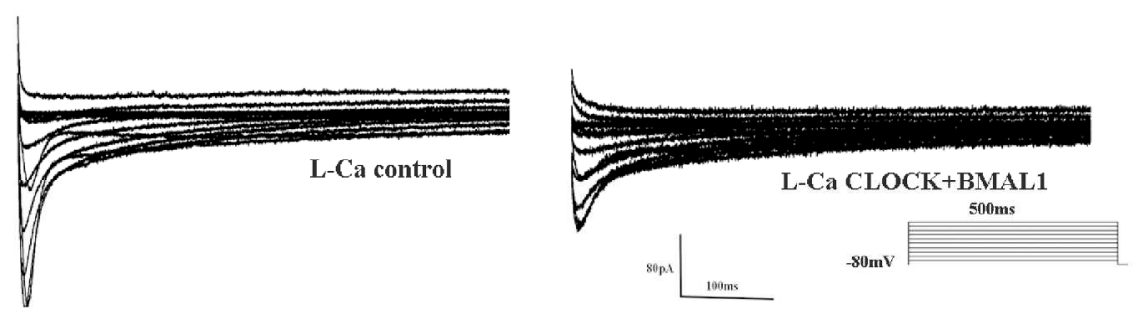

B

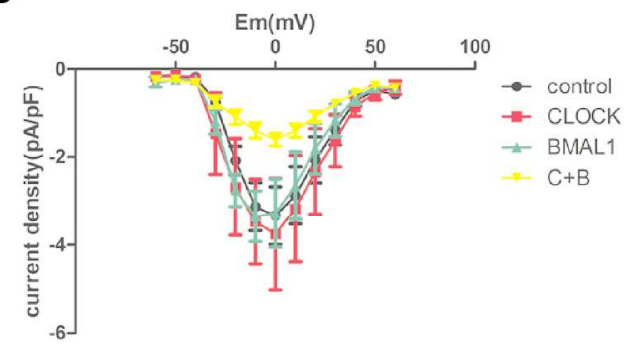

C

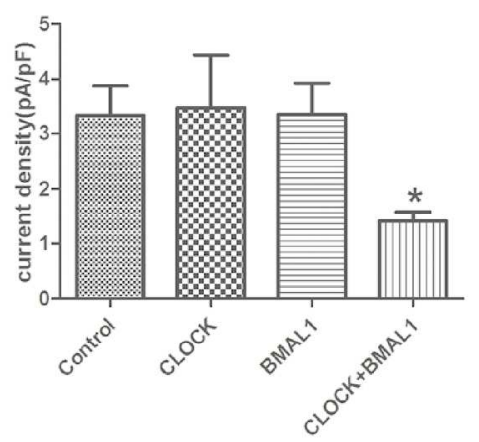

Figure4. L-type calcium currents were reduced in the CLOCK-BMAL1 over-expressed cardiomyocytes. $185 \times 146 \mathrm{~mm}(300 \times 300 \mathrm{DPI})$ 

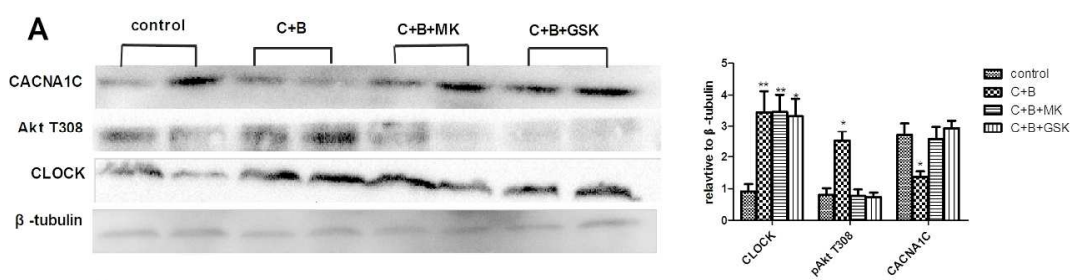

B
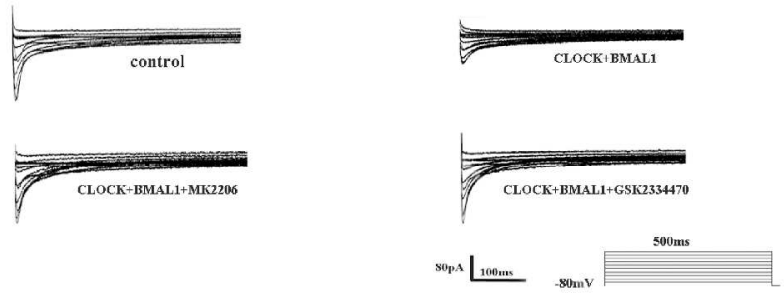

C
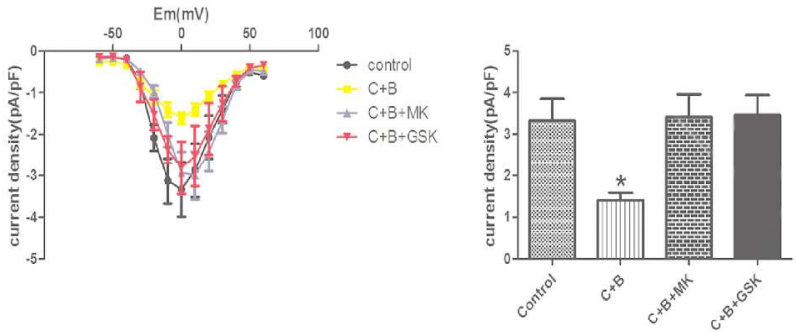

D
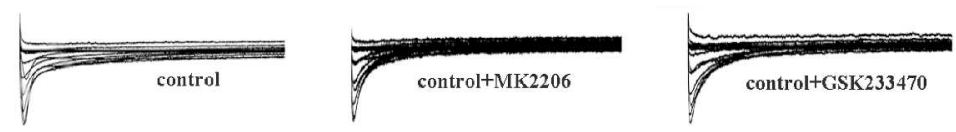
${ }_{80 \mathrm{pA}} \bigsqcup_{100 \mathrm{~ms}}$

somv

$E$
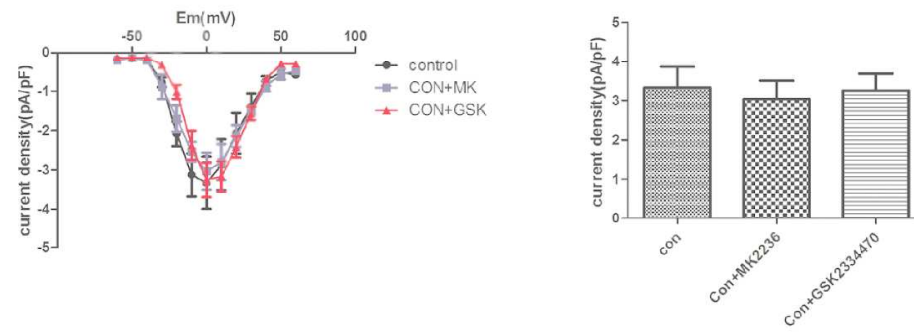

Figure5. The inhibitory effects of CLOCK-BMAL1 on the L-type calcium currents were abolished by Akt inhibitor MK2206 or PDK1 inhibitor GSK2334470.

$200 \times 282 \mathrm{~mm}(300 \times 300 \mathrm{DPI})$ 